\title{
Gut microbiome in systemic sclerosis: a potential therapeutic target
}

\author{
Beata Polkowska-Pruszyńska ${ }^{1}$, Agnieszka Gerkowicz ${ }^{1}$, Karol Rawicz-Pruszyński² ${ }^{2}$, Dorota Krasowska ${ }^{1}$
}

${ }^{1}$ Chair and Department of Dermatology, Venereology and Paediatric Dermatology, Medical University of Lublin, Lublin, Poland ${ }^{2}$ Department of Surgical Oncology, Medical University of Lublin, Lublin, Poland

Adv Dermatol Allergol 2022; XXXIX (1): 101-109

DOI: https://doi.org/10.5114/ada.2020.101468

\begin{abstract}
Introduction: Systemic sclerosis (SSc) is a chronic, connective tissue disease characterized by inflammation, fibrosis and microcirculation disturbances. Gastrointestinal involvement and impaired gut motility observed in SSc promotes the small intestinal bacterial overgrowth (SIBO) defined as the increase in the number of bacteria to over $10^{5} \mathrm{CFU} / \mathrm{ml}$ or as the presence of atypical flora.

Aim: To investigate the prevalence and characteristics of SIBO and to assess the efficacy of rifaximin in SIBO treatment in SSc patients.

Material and methods: 40 SSc patients and 39 healthy individuals were enrolled in the study. All subjects completed UCLA SCTC GIT 2.0 questionnaire and query for gastrointestinal symptoms. The presence of SIBO was assessed by the lactulose hydrogen breath test (LHBT). Patients with SIBO received $1200 \mathrm{mg}$ rifaximin daily for 10 days. The same diagnostic procedure was performed after completed treatment in order to evaluate SIBO eradication.

Results: The prevalence of SIBO was higher in SSc patients compared with the control group (47.5\% vs. $12.8 \%$; $p=0.0008$ ). SIBO eradication after rifaximin treatment was successful in $73.3 \%$ of SSc patients.

Conclusions: These data suggest that SIBO occurs more frequently in SSc patients than in controls. Eradication therapy with rifaximin is associated with satisfactory results and a high safety profile.
\end{abstract}

Key words: small intestinal bacterial overgrowth, small intestinal bacterial overgrowth, systemic sclerosis, rifaximin.

\section{Introduction}

Systemic sclerosis (SSc) is an autoimmune chronic, connective tissue disease characterised by vasculopathy, progressive inflammation and fibrosis of the skin and internal organs [1]. The standardized mortality ratio for SSc patients is 3.5 as compared to the control group $[2,3]$. EUSTAR research assessed that of the SSc-related deaths, 35\% were attributed to pulmonary fibrosis, 26\% to pulmonary arterial hypertension (PAH) and $26 \%$ to cardiac causes [4]. However, one of the studies additionally placed gastrointestinal complications next to PAH as the leading cause of SSc-related deaths [5]. Indeed, approximately $90 \%$ of SSc patients present variously intensified fibrosis in the gastrointestinal tract (GIT) [6]. The pathogenetic background underlying gastrointestinal manifestations involves endothelial cells damage, vasculopathy, release of cytokines, $\mathrm{T}$ and B-lymphocytes activation and production of autoantibodies against gastrointestinal smooth muscles muscarinic receptors $[7,8]$.
The small intestine involvement in SSc is estimated at a prevalence of 23-88\% [9-11]. Hypomotility, the luminal content stasis and frequent proton pump inhibitors intake in SSc patients potentiate the possibility of bacterial colonisation and the development of small intestinal bacterial overgrowth syndrome (SIBO). SIBO is defined as the increase exceeding $10^{5}$ colony forming units of bacteria per $\mathrm{ml}$ of jejunal juice or as the presence of atypical flora. In the course of the disease, symptoms such as bloating, flatulence, diarrhoea or even steatorrhea, fatsoluble vitamins deficiency symptoms, malignant anaemia, malnutrition, hypoproteinaemia symptoms and systemic disorders may be encountered [9, 12-14].

The lack of standardization of available diagnostic tests makes SIBO diagnosis a clinical challenge. Regardless of high sensitivity, small intestinal aspirate culture is only a partially validated diagnostic procedure. The costs, invasiveness, complexity and low accessibility are also discouraging factors. In comparison with duodenal aspirate

Address for correspondence: Beata Polkowska-Pruszyńska, Chair and Department of Dermatology, Venerology and Paediatric Dermatology, Medical University of Lublin, 16 Staszica St, 20-081 Lublin, Poland, phone: +48 815323 647, e-mail: polkowska.beata@gmail.com Received: 3.10.2020, accepted: 30.10.2020. 
culture, hydrogen breath tests have lower sensitivity but good specificity for detection of SIBO. Therefore, hydrogen breath tests remain an attractive alternative and should be used as a first line diagnostic procedure $[15,16]$. Furthermore, a lactulose hydrogen breath test proved to be useful in lactose malabsorption detection, which quite commonly occurs in SSc patients, who may especially take advantage of lactose intake reduction [17]. Therapeutic options in SIBO include metronidazole, amoxicillin with clavulanic acid, co-trimoxazole, ciprofloxacin, norfloxacin, rifaximin, octreotide [18]. There are only few studies reporting SIBO prevalence treatment efficacy in SSc patients. Only 4 studies assessing SIBO prevalence in SSc included control groups and only 6 studies evaluated the eradication rate of different therapies [18]. So far, no reports have been established for the population of SSc patients in comparison to healthy controls in Eastern European countries.

\section{Aim}

The aim of the present prospective study was to (1) assess the prevalence and characteristics of small intestinal bacterial overgrowth syndrome in SSc patients, (2) evaluate the eradication rate for rifaximin in the therapy of SIBO in SSc patients and (3) compare certain variables between the study and control groups.

\section{Material and methods}

\section{Patients}

From December 2018 to February 2020, 40 consecutive patients diagnosed with SSc and 39 healthy individuals were enrolled in the study in the Department of Dermatology, Venerology and Paediatric Dermatology, Medical University of Lublin, Poland. The diagnosis of SSc was based on the 2013 classification criteria for systemic sclerosis by ACR/EULAR $[19,20]$. Ethical approval was obtained from the local ethical committee (number KE-0254/238/2018) and informed consent was attained from all patients. The study cohort consisted of 39 (97.5\%) women and 1 (2.5\%) man with a median age of 62 years (range: 34-78), weight of 65 kilograms (range: 50-99) and body mass index (BMI) $24.9 \mathrm{~kg} / \mathrm{m}^{2}$ (range: 19.8-34). The median duration of the disease was 10.5 years (range: $1-30)$. The control group of 39 healthy individuals consisted of 36 (92.3\%) women and $3(7.7 \%)$ men with a median age of 61 years (range: $33-77)$, weight of $69 \mathrm{~kg}$ (range: 53-110) and BMI $26.4 \mathrm{~kg} / \mathrm{m}^{2}$ (range: 19-38.4). All individuals in the control group confirmed they had no pre-existing chronic diseases nor any gastrointestinal comorbidities diagnosed. The study and control groups were comparable in terms of sex, age and weight. All patients' characteristics are presented in Table 1.

\section{Gastrointestinal symptoms}

Before undergoing the lactulose hydrogen breath test (LHBT) all patients and healthy controls were in- terviewed regarding the presence of gastrointestinal symptoms including: diarrhoea, constipation, abdominal pain/discomfort, flatulence, abdominal tenderness, nausea, tenesmus, general illness and reflux. Additionally, all study participants completed the UCLA Scleroderma Clinical Trial Consortium GIT 2.0 (UCLA SCTC GIT 2.0) questionnaire, which includes 7 scales (reflux, distention/bloating, diarrhoea, faecal soiling, constipation, emotional well-being, and social functioning) to assess gastrointestinal symptoms severity in SSc. All scales are scored from 0.00 to 3.00 except the diarrhoea and constipation (range $0.00-2.00$ and $0.00-2.50$, respectively). The questionnaire provides a total score by summation of all scales (except constipation) and ranges from 0.00-2.83 [21]. Gastrointestinal symptoms were compared between SSC patients with and without SIBO diagnosis and the control group.

\section{Lactulose hydrogen breath test (LHBT)}

All participants were properly instructed before LHBT and were not allowed to take antibiotics or undergo colonoscopy or fluoroscopy 4 weeks before the test. One week prior to LHBT, patients avoided thickeners or stool relaxants, vitamins, supplements containing fructose as a flavouring substance or lactose as a filler, fibre supplements, prebiotics and probiotics, proton pump inhibitors and $\mathrm{H} 2$ receptor antagonists. Food products likely to generate hydrogen were not allowed 3 days before LHBT. LHBT was performed after an overnight fast of minimum 12 h. Expired air sample was analysed using Gastrolyzer (Bedfont, UK). Basal air sample was rated at fasting, and then after oral administration of lactulose $(20 \mathrm{~g}$ in $200 \mathrm{ml}$ of sterile water) at intervals of 20 min during the period of 3 h. A 20 parts per million (ppm) increase of expired hydrogen between the maximum reading and the fasting value was considered as a positive result. The results were compared between SSc patients with and without $\mathrm{SIBO}$ diagnosis and the control group.

\section{Biochemical, clinical and demographic characteristic of SSc patients}

The following laboratory findings were compared between SSc patients with and without SIBO diagnosis: erythrocyte sedimentation rate (ESR; $\mathrm{mm} / \mathrm{h}$ ), C-reactive protein $(\mathrm{mg} / \mathrm{l})$, total protein $(\mathrm{g} / \mathrm{dl})$, albumin (\% of total protein), C3 complement (g/l), C4 complement (g/l), creatine kinase (CK, IU/l), rheumatoid factor (IU/ml), NTproBNP $(\mathrm{pg} / \mathrm{ml})$, leukocytes $(\mathrm{K} / \mu \mathrm{l})$; and antibody status: anti-centromere (ACA), anti-topoisomerase I (anti-Scl70) and anti-RNA polymerase III antibodies. Furthermore, $\mathrm{SIBO}+/$-patients were compared in terms of median age; weight; BMI; SSc duration; SSc subsets; prevalence of digital ulcers/pitting scars; pulmonary involvement; joint involvement; Raynaud's phenomenon presence, changes in capillaroscopy and gastrointestinal comorbidities. 
Table 1. Characteristics of the study and control groups including SIBO presence

\begin{tabular}{|c|c|c|c|c|}
\hline Variable & Group & Absence of SIBO & Presence of SIBO & $P$-value \\
\hline \multicolumn{5}{|l|}{ Characteristics of the study group including SIBO presence: } \\
\hline \multicolumn{4}{|l|}{ Gender: } & \multirow[t]{3}{*}{0.9596} \\
\hline Female & $39(97.5 \%)$ & $20(95.2 \%)$ & 19 (100\%) & \\
\hline Male & $1(2.5 \%)$ & $1(4.8 \%)$ & - & \\
\hline \multicolumn{4}{|l|}{ Disease subtype: } & \multirow[t]{3}{*}{0.5153} \\
\hline dcSCC & $6(15.4 \%)$ & $2(9.52 \%)$ & $4(22.2 \%)$ & \\
\hline IcSCC & $33(84.6 \%)$ & $19(90.48 \%)$ & $14(77.8 \%)$ & \\
\hline \multicolumn{4}{|l|}{ Antibodies: } & \multirow[t]{5}{*}{0.7338} \\
\hline ACA & $22(59.5 \%)$ & $12(60 \%)$ & $10(58.8 \%)$ & \\
\hline Scl-70 & $12(32.4 \%)$ & $7(35 \%)$ & $5(29.4 \%)$ & \\
\hline Not specified & $1(2.7 \%)$ & - & $1(5.9 \%)$ & \\
\hline ACA/Scl-70 & $2(5.4 \%)$ & $1(5 \%)$ & $1(5.9 \%)$ & \\
\hline \multicolumn{4}{|l|}{ Interstitial lung disease: } & \multirow[t]{4}{*}{0.9406} \\
\hline No & $14(35 \%)$ & $7(33.3 \%)$ & $7(36.8 \%)$ & \\
\hline Yes & $18(45 \%)$ & $10(47.6 \%)$ & $8(42.1 \%)$ & \\
\hline In diagnosis & $8(20 \%)$ & $4(19.1 \%)$ & $4(21.1 \%)$ & \\
\hline \multicolumn{5}{|l|}{ Digital ulcers/pitting scars: } \\
\hline No & $19(47.5 \%)$ & $8(38.1 \%)$ & $11(57.9 \%)$ & \multirow[t]{2}{*}{0.3497} \\
\hline Yes & $21(52.5 \%)$ & $13(61.9 \%)$ & $8(42.1 \%)$ & \\
\hline \multicolumn{5}{|l|}{ Gastrointestinal comorbidities: } \\
\hline No & $24(60 \%)$ & $12(57.1 \%)$ & $12(63.2 \%)$ & \multirow[t]{2}{*}{0.9485} \\
\hline Yes & $16(40 \%)$ & $9(42.9 \%)$ & $7(36.8 \%)$ & \\
\hline \multicolumn{5}{|l|}{ Gastrointestinal comorbidities: } \\
\hline GERD & $5(31.3 \%)$ & $3(33.3 \%)$ & $2(28.6 \%)$ & \multirow[t]{3}{*}{0.9539} \\
\hline Oesophageal hiatal hernia & $7(43.7 \%)$ & $4(44.4 \%)$ & $3(42.9 \%)$ & \\
\hline Others (including mechanical bowel obstruction) & $4(25 \%)$ & $2(22.2 \%)$ & $2(28.6 \%)$ & \\
\hline \multicolumn{5}{|l|}{ Joint pain: } \\
\hline No & $12(30.8 \%)$ & $5(25 \%)$ & $7(36.8 \%)$ & \multirow[t]{2}{*}{0.6499} \\
\hline Yes & $27(69.2 \%)$ & $15(75 \%)$ & $12(63.2 \%)$ & \\
\hline \multicolumn{5}{|l|}{ Raynaud phenomenon: } \\
\hline Yes & $40(100 \%)$ & $21(100 \%)$ & $19(100 \%)$ & - \\
\hline \multicolumn{5}{|l|}{ Changes in capillaroscopy: } \\
\hline No & $1(2.7 \%)$ & - & $1(5.6 \%)$ & \multirow[t]{2}{*}{0.9781} \\
\hline Yes & $36(97.3 \%)$ & $19(100 \%)$ & $17(94.4 \%)$ & \\
\hline \multicolumn{5}{|l|}{ SIBO presence: } \\
\hline No & $21(52.5 \%)$ & $21(100 \%)$ & - & \multirow[t]{2}{*}{-} \\
\hline Yes & $19(47.5 \%)$ & - & $19(100 \%)$ & \\
\hline \multicolumn{5}{|l|}{ SIBO eradication: } \\
\hline No & $4(26.7 \%)$ & - & $4(26.7 \%)$ & \multirow[t]{2}{*}{-} \\
\hline Yes & $11(73.3 \%)$ & & $11(73.3 \%)$ & \\
\hline Characteristics of the control group including SIBO pr & & & & \\
\hline Gender: & & & & \\
\hline Female & $36(92.3 \%)$ & $31(91.2 \%)$ & $5(100 \%)$ & 0.8357 \\
\hline Male & $3(7.7 \%)$ & $3(8.8 \%)$ & - & \\
\hline
\end{tabular}

SIBO - small intestinal bacterial overgrowth, dcSCC - diffuse cutaneous SSC type, ICSCC - limited cutaneous, SSC type, ACA - anticentromere antibodies, $\mathrm{SCl}-70$ - antibodies against topoisomerase I, GERD - gastroesophageal reflux disease. 


\section{Eradication of SIBO}

All patients diagnosed with SIBO were administered rifaximin $400 \mathrm{mg} 3$ times daily for the period of 10 consecutive days. One month after the therapy the patients underwent LHBT to determine the eradication of SIBO. The patients were also screened for the presence of gastrointestinal symptoms. We measured the eradication rate of SIBO after rifaximin treatment as well as GIT symptoms.

\section{Statistical analysis}

All analyses were performed using MedCalc 15.8 (MedCalc Software, Ostend, Belgium). For group comparison involving binary data (or for several subgroups), we used either the $\chi^{2}$ test or Fisher's exact test. Comparisons involving continuous data were performed using: 1) Student's test when distribution of variables was normal; and 2) Mann-Whitney test in other cases. The Kruskal-Wallis test was used for multiple comparisons of continuous data. For comparisons of data collected in subsequent time-points, we used the Wilcoxon test. The results were regarded as significant when the $p$-value was less than 0.05 .

\section{Results}

\section{Prevalence of SIBO}

In the study group, 19 (47.5\%) patients were diagnosed with SIBO, whereas in the control group SIBO was diagnosed in 5 cases $(12.8 \%$; $p=0.0008)$.

\section{Gastrointestinal symptoms}

All gastrointestinal symptoms were more frequent in the study group when compared to the control group, in terms of abdominal pain or discomfort ( $85 \%$ vs. $48.7 \%$, $p=0.0014)$ and general illness (90\% vs. $41 \%, p<0.0001$ ); the results were significant. All gastrointestinal complaints of the patients were observed less often during the second assessment after SIBO treatment in comparison to the first measurement prior to the therapy, in case of the constipation the result was statistically significant (57.1\% vs. $87.5 \%, p=0.0412$ ). Diarrhoea, flatulence, abdominal tenderness, tenesmus and reflux were more common in SSc patients with SIBO diagnosis. Other gastrointestinal complaints of the patients are summarized in Table 2.

In the UCLA GIT 2.0 questionnaire, reflux was significantly more intensified in the study group (0.25 vs. 0.125 ,

Table 2. The prevalence of gastrointestinal symptoms in control and study groups depending on SIBO presence, eradication and the time of LHBT measurement

\begin{tabular}{|c|c|c|c|c|c|c|c|c|c|c|c|c|}
\hline \multirow[t]{3}{*}{ Symptom } & \multirow{3}{*}{$\begin{array}{l}\text { Control } \\
\text { group }\end{array}$} & \multirow{3}{*}{$\begin{array}{l}\text { Study } \\
\text { group }\end{array}$} & \multirow[t]{3}{*}{$P$-value } & \multicolumn{9}{|c|}{ Study group } \\
\hline & & & & \multicolumn{2}{|c|}{ Measurement } & \multirow[t]{2}{*}{$P$-value } & \multicolumn{2}{|c|}{ SIBO } & \multirow[t]{2}{*}{$P$-value } & \multicolumn{2}{|c|}{ Eradication } & \multirow[t]{2}{*}{$P$-value } \\
\hline & & & & I & II & & Absent & Present & & No & Yes & \\
\hline Diarrhoea & $26(66.7 \%)$ & $30(75 \%)$ & 0.5704 & $30(75 \%)$ & 7 (50\%) & 0.1618 & $14(66.7 \%)$ & $16(84.2 \%)$ & 0.3607 & $2(50 \%)$ & $5(50 \%)$ & 0.5541 \\
\hline Constipation & 28 (71.8\%) & $35(87.5 \%)$ & 0.1452 & 35 (87.5\%) & $8(57.1 \%)$ & 0.0412 & $19(90.5 \%)$ & $16(84.2 \%)$ & 0.9047 & $2(50 \%)$ & $6(60 \%)$ & 0.7978 \\
\hline $\begin{array}{l}\text { Abdominal } \\
\text { pain/ } \\
\text { discomfort }\end{array}$ & 19 (48.7\%) & $34(85 \%)$ & 0.0014 & $34(85 \%)$ & $9(64.3 \%)$ & 0.2038 & $19(90.5 \%)$ & $15(79 \%)$ & 0.5644 & $3(75 \%)$ & $6(60 \%)$ & 0.9297 \\
\hline Flatulence & $27(69.2 \%)$ & $33(82.5 \%)$ & 0.2643 & $33(82.5 \%)$ & $12(85.7 \%)$ & 0.8896 & $16(76.2 \%)$ & 17 (89.5\%) & 0.4918 & $4(100 \%)$ & $8(80 \%)$ & 0.9039 \\
\hline $\begin{array}{l}\text { Abdominal } \\
\text { tenderness }\end{array}$ & $12(30.8 \%)$ & $14(35 \%)$ & 0.8724 & $14(35 \%)$ & $6(42.9 \%)$ & 0.8396 & $4(19 \%)$ & $10(52.6 \%)$ & 0.0585 & $2(50 \%)$ & $4(40 \%)$ & 0.7978 \\
\hline Nausea & $14(35.9 \%)$ & $20(50 \%)$ & 0.2991 & $20(50 \%)$ & $6(42.9 \%)$ & 0.8811 & $10(47.6 \%)$ & $10(52.6 \%)$ & 1.0000 & $1(25 \%)$ & $5(50 \%)$ & 0.7978 \\
\hline Vomiting & $21(53.9 \%)$ & $13(32.5 \%)$ & 0.0913 & $13(32.5 \%)$ & $6(42.9 \%)$ & 0.7089 & $8(38.1 \%)$ & $5(26.3 \%)$ & 0.6482 & $1(25 \%)$ & $5(50 \%)$ & 0.7978 \\
\hline Dysuria & $7(18 \%)$ & $7(17.5 \%)$ & 0.7500 & $7(17.5 \%)$ & $3(21.4 \%)$ & 0.9410 & $3(14.3 \%)$ & $4(21.1 \%)$ & 0.8841 & $1(25 \%)$ & $2(20 \%)$ & 0.6066 \\
\hline Tenesmus & $24(61.55 \%)$ & $27(67.5 \%)$ & 0.7500 & $27(67.5 \%)$ & $8(57.1 \%)$ & 0.7089 & $13(61.9 \%)$ & $14(73.7 \%)$ & 0.6482 & $2(50 \%)$ & $6(60 \%)$ & 0.7978 \\
\hline Fever & $14(37.8 \%)$ & $14(35 \%)$ & 0.9828 & $14(35 \%)$ & $5(35.7 \%)$ & 0.7818 & 7 (33.3\%) & 7 (36.8\%) & 0.9207 & $1(25 \%)$ & $4(40 \%)$ & 0.9297 \\
\hline $\begin{array}{l}\text { General } \\
\text { illness }\end{array}$ & $16(41 \%)$ & $36(90 \%)$ & $<0.0001$ & $36(90 \%)$ & $10(71.4 \%)$ & 0.2126 & $18(85.7 \%)$ & $18(94.7 \%)$ & 0.6729 & $3(75 \%)$ & $7(70 \%)$ & 0.6400 \\
\hline Reflux & $18(46.1 \%)$ & $27(67.5 \%)$ & 0.0913 & $27(67.5 \%)$ & $7(50 \%)$ & 0.3978 & $12(57.1 \%)$ & $15(79 \%)$ & 0.2575 & $2(50 \%)$ & $5(50 \%)$ & 0.5541 \\
\hline Dysphagia & 15 (38.5\%) & $16(40 \%)$ & 0.9280 & $16(40 \%)$ & $6(42.9 \%)$ & 0.8976 & $6(28.6 \%)$ & $10(52.6 \%)$ & 0.2195 & $2(50 \%)$ & $4(40 \%)$ & 0.7978 \\
\hline $\begin{array}{l}\text { Early } \\
\text { satiety }\end{array}$ & $21(53.8 \%)$ & $23(57.5 \%)$ & 0.9201 & $23(57.5 \%)$ & $7(50 \%)$ & 0.8622 & $9(42.9 \%)$ & $14(73.7 \%)$ & 0.0991 & $1(25 \%)$ & $6(60 \%)$ & 0.5541 \\
\hline
\end{tabular}

SIBO - small intestinal bacterial overgrowth, LHBT - lactulose hydrogen breathing test. 
Table 3. The UCLA GIT 2.0 questionnaire results

\begin{tabular}{|c|c|c|c|c|c|c|c|c|c|c|c|c|}
\hline \multirow[t]{3}{*}{ Symptom } & \multirow{3}{*}{$\begin{array}{l}\text { Control } \\
\text { group }\end{array}$} & \multirow{3}{*}{$\begin{array}{l}\text { Study } \\
\text { group }\end{array}$} & \multirow[t]{3}{*}{$P$-value } & \multicolumn{9}{|c|}{ Study group } \\
\hline & & & & \multicolumn{2}{|c|}{ Measurement } & \multirow[t]{2}{*}{$P$-value } & \multicolumn{2}{|c|}{ SIBO } & \multirow[t]{2}{*}{$P$-value } & \multicolumn{2}{|c|}{ Eradication } & \multirow[t]{2}{*}{$P$-value } \\
\hline & & & & I & II & & Absent & Present & & No & Yes & \\
\hline Reflux & 0.00 & 0.25 & 0.0001 & 0.25 & 0.25 & 0.7869 & 0.25 & 0.25 & 0.1865 & 0.25 & 0.25 & 0.8946 \\
\hline Flatulence & 0.25 & 0.62 & 0.0048 & 0.50 & 0.50 & 0.8501 & 0.50 & 0.75 & 0.2965 & 0.50 & 0.75 & 0.2848 \\
\hline Faecal soiling & 0.00 & 0.00 & 0.0014 & 0.00 & 0.00 & NR & 0.00 & 0.00 & NR & 0.00 & 0.00 & NR \\
\hline Diarrhoea & 0.00 & 0.00 & 0.5160 & 0.00 & 0.00 & 1.0000 & 0.00 & 0.00 & 0.3894 & 0.00 & 0.00 & 0.1106 \\
\hline Social functioning & 0.00 & 0.16 & 0.6182 & 0.33 & 0.16 & 0.5186 & 0.16 & 0.33 & 0.1659 & 0.16 & 0.33 & 0.6333 \\
\hline Emotional well-being & 0.08 & 0.11 & 0.9056 & 0.16 & 0.00 & 0.1309 & 0.00 & 0.16 & 0.1274 & 0.05 & 0.22 & 0.4575 \\
\hline Constipation & 0.50 & 0.37 & 0.7129 & 0.25 & 0.25 & 0.9219 & 0.25 & 0.50 & 0.9331 & 0.37 & 0.25 & 0.6844 \\
\hline Total score & 0.31 & 0.19 & 0.3512 & 0.25 & 0.19 & 0.3054 & 0.17 & 0.35 & 0.1711 & 0.17 & 0.38 & 0.3603 \\
\hline
\end{tabular}

UCLA SCTC GIT 2.0 - UCLA Scleroderma Clinical Trial Consortium GIT 2.0.

$p=0.0164)$. Additionally, flatulence, social functioning impairment, emotional well-being decrease constipation intensity, which was higher in the study group in comparison to the control group. The patients in the study group noted higher total score values (0.19 vs. 0.16). After the rifaximin treatment we observed improvement in the UCLA GIT 2.0 questionnaire in the terms of social functioning, emotional well-being, and total score. The SSc patients diagnosed with SIBO were experiencing more commonly flatulence, constipation, decreased social functioning and emotional well-being as well as higher total score values. Furthermore, reflux complaints were more severe in SSC patients with $\mathrm{SIBO}$ diagnosis than in SIBO positive control group cases ( $p=0.0065$ ). The assessment of other UCLA GIT 2.0 parameters can be found in Table 3 .

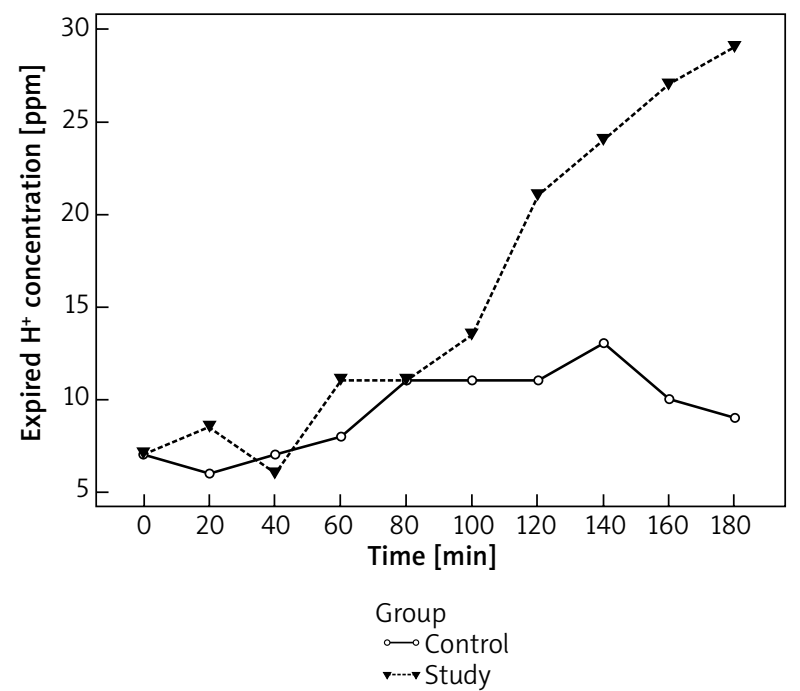

Figure 1. Comparison of the expired hydrogen value at successive time points in the control and study groups

\section{Lactulose hydrogen breath test (LHBT)}

We observed significantly elevated values of expired hydrogen in the study group in comparison with the control group after 60 (11 vs. $6 ; p=0.0411), 120$, (21 vs. 11; $p=0.0162$ ), 140 (24 vs. $13 ; p=0.0012$ ), 160 (27 vs. 10; $p<0.0001)$ and 180 (29 vs. 9; $p=0.0004$ ) min from the beginning of the test (Figure 1). Similar differences were observed when the first and second LHBT measurements were compared. Significantly higher expired hydrogen values were noted during the first measurement in comparison to the second measurement, approximately after 120 (39 vs. $10 ; p=0.0181$ ), 140 (42 vs. $14 ; p=0.0034$ ), 160 (46 vs. $19 ; p=0.0054$ ) and 180 (43 vs. $16 ; p=0.0067$ ) $\mathrm{min}$ from the beginning of the test (Figure 2). Detailed characteristics of LHBT measurements are presented in Table 4.

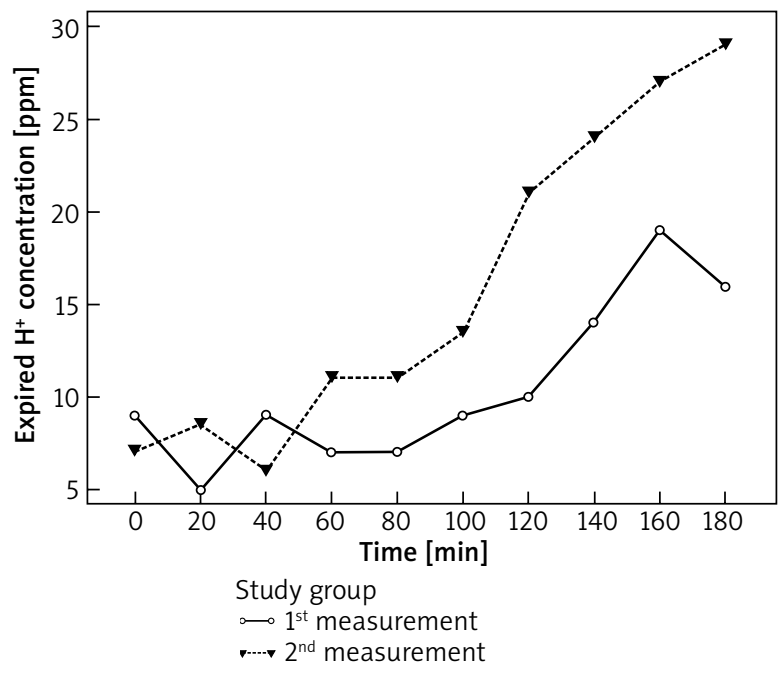

Figure 2. Comparison of the expired hydrogen value at subsequent time points in the study group after rifaximin therapy 
Table 4. Characteristics and comparison of the measurement of exhaled hydrogen between the control and treated group (including before and after treatment)

\begin{tabular}{|c|c|c|c|c|c|c|c|c|c|c|c|}
\hline \multirow{3}{*}{$\begin{array}{l}\text { Measurement } \\
\text { time [min] }\end{array}$} & \multirow{2}{*}{\multicolumn{3}{|c|}{ Control group }} & \multicolumn{6}{|c|}{ Study group } & \multirow[t]{3}{*}{$P$-value* } & \multirow[t]{3}{*}{$P$-value ${ }^{\star \star}$} \\
\hline & & & & \multicolumn{3}{|c|}{$\begin{array}{l}1^{\text {st }} \text { measurement } \\
\text { (before treatment) }\end{array}$} & \multicolumn{3}{|c|}{$\begin{array}{l}2^{\text {nd }} \text { measurement } \\
\text { (after treatment) }\end{array}$} & & \\
\hline & $N$ & $\mathrm{Me}$ & Range & $N$ & Me & Range & $N$ & $\mathrm{Me}$ & Range & & \\
\hline 0 & 39 & 7.0 & $0.0-58.0$ & $\begin{array}{l}40 \\
(15)\end{array}$ & $\begin{array}{l}7.0 \\
(5.0)\end{array}$ & $0.0-32.0$ & $\begin{array}{l}15 \\
(15)\end{array}$ & $\begin{array}{c}9.0 \\
(9.0)\end{array}$ & $1.0-24.0$ & 0.8132 & 0.6387 \\
\hline 20 & 39 & 6.0 & $0.0-63.0$ & $\begin{array}{r}40 \\
(15)\end{array}$ & $\begin{array}{c}8.5 \\
(8.0)\end{array}$ & $0.0-33.0$ & $\begin{array}{c}15 \\
(15)\end{array}$ & $\begin{array}{c}5.0 \\
(5.0)\end{array}$ & $1.0-24.0$ & 0.3821 & 0.5830 \\
\hline 40 & 39 & 7.0 & $1.0-54.0$ & $\begin{array}{l}40 \\
(15)\end{array}$ & $\begin{array}{c}6.0 \\
(6.0)\end{array}$ & $0.0-36.0$ & 15 & $\begin{array}{c}9.0 \\
(9.0)\end{array}$ & $1.0-24.0$ & 0.8674 & 0.7197 \\
\hline 60 & 39 & 8.0 & $0.0-49.0$ & $\begin{array}{r}40 \\
(15)\end{array}$ & $\begin{array}{c}11.0 \\
(11.0)\end{array}$ & $0.0-54.0$ & 15 & $\begin{array}{c}7.0 \\
(7.0)\end{array}$ & $1.0-31.0$ & 0.1562 & 0.2524 \\
\hline 80 & 39 & 11.0 & $0.0-56.0$ & $\begin{array}{r}40 \\
(15)\end{array}$ & $\begin{array}{c}11.0 \\
(11.0)\end{array}$ & $0.0-192.0$ & 15 & $\begin{array}{c}7.0 \\
(7.0)\end{array}$ & $1.0-28.0$ & 0.3436 & 0.1876 \\
\hline 100 & 39 & 11.0 & $1.0-77.0$ & $\begin{array}{c}40 \\
(15)\end{array}$ & $\begin{array}{c}13.5 \\
(19.0)\end{array}$ & 0.0-198.0 & 15 & $\begin{array}{c}9.0 \\
(9.0)\end{array}$ & $1.0-62.0$ & 0.2491 & 0.1688 \\
\hline 120 & 39 & 11.0 & $0.0-81.0$ & $\begin{array}{l}40 \\
(15)\end{array}$ & $\begin{array}{l}21.0 \\
(39.0)\end{array}$ & $0.0-160.0$ & 15 & $\begin{array}{l}10.0 \\
(10.0)\end{array}$ & $1.0-75.0$ & 0.0162 & 0.0181 \\
\hline 140 & 39 & 13.0 & $0.0-82.0$ & $\begin{array}{c}40 \\
(15)\end{array}$ & $\begin{array}{l}24.0 \\
(42.0)\end{array}$ & $0.0-134.0$ & 15 & $\begin{array}{c}14.0 \\
(14.0)\end{array}$ & $1.0-70.0$ & 0.0012 & 0.0034 \\
\hline 160 & 23 & 10.0 & $1.0-66.0$ & $\begin{array}{c}40 \\
(15)\end{array}$ & $\begin{array}{l}27.0 \\
(46.0)\end{array}$ & $0.0-134.0$ & 15 & $\begin{array}{c}19.0 \\
(19.0)\end{array}$ & $1.0-68.0$ & $<0.0001$ & 0.0054 \\
\hline 180 & 23 & 9.0 & $2.0-61.0$ & $\begin{array}{l}40 \\
(15)\end{array}$ & $\begin{array}{c}29.0 \\
(43.0)\end{array}$ & $0.0-197.0$ & 15 & $\begin{array}{l}16.0 \\
(16.0)\end{array}$ & $1.0-73.0$ & 0.0004 & 0.0067 \\
\hline
\end{tabular}

"Control group vs. study group (15t measurement) (U-Mann-Whitney test), "study group: 1 measurement vs. $2^{\text {nd }}$ measurement (Wilcoxon test). Values in brackets „()" show results of Wilcoxon test (paired).

Biochemical, clinical and demographic comparison of SSc patients depending on SIBO diagnosis

Significantly higher albumin values were observed in SSc patients diagnosed with SIBO in comparison with SSc patients without SIBO diagnosis (medians: 58.5 vs. 52.3; $p=0.0233$ ). Detailed characteristics and comparison of selected demographic, clinical and biochemical variables in SSc patients are presented in Table 5.

\section{Eradication of SIBO in SSc patients}

SIBO therapy with rifaximin was well tolerated by all SSC patients. One month after the therapy the patients underwent LHBT to determine SIBO eradication. Out of 19 SSc patients with SIBO diagnosis 15 patients attended the check-up LHBT. Four patients were lost from the follow up due to illness or withdrawal of willingness to participate in the study. In the final results concerning eradication of the disease we did not include healthy controls diagnosed with SIBO as the priority of our research was assessing SIBO eradication in the course of SSc. Furthermore, in the control group, SIBO was diagnosed in only 5 cases, which did not comprise a representative group. Eradication of SIBO was achieved in $73.3 \%$ of the patients. We observed a significant decrease in constipation severity after SIBO eradication ( 0.87 vs. $0.25, p=0.0147$ ).

\section{Discussion}

SSc patients are at high risk of developing gastrointestinal malfunctions [10, 22]. Even $90 \%$ of them report gastrointestinal complaints, which may be associated with relevant morbidity and mortality $[5,6]$. The small intestine is the second most common affected organ in GIT causing signs such as nausea, vomiting, bloating, diarrhoea and malnutrition. Moreover, SSc patients commonly suffer from reflux, dysphagia or constipation [12]. Our study showed an increased incidence of all examined gastrointestinal symptoms in SSc patients in comparison with controls.

Pathological changes observed within the small bowel may lead to the imbalance of gastrointestinal defence mechanisms predisposing to small intestinal bacterial overgrowth (SIBO) [23-33]. Different studies show the presence of SIBO in 18-55\% of SSc patients [26-32]. Our data remain in agreement with these results - the presence of SIBO was found in $47.5 \%$ of SSC patients and was significantly more frequent than in the healthy controls, where SIBO diagnosis concerned $12.8 \%$ of cases. According to the recent studies, SIBO is diagnosed in even up to $20 \%$ of healthy study controls [24, 28, 30, 34-40]. 
Table 5. Characteristics and comparison of selected demographic, clinical and biochemical variables in SSc patients without or with SIBO diagnosis

\begin{tabular}{|c|c|c|c|c|c|c|c|}
\hline \multirow[t]{2}{*}{ Variable } & \multicolumn{3}{|c|}{ SIBO absence } & \multicolumn{3}{|c|}{ SIBO presence } & \multirow[t]{2}{*}{$P$-value } \\
\hline & $\operatorname{Me}(n)$ & Min. & Max. & $\operatorname{Me}(n)$ & Min. & Max. & \\
\hline Age [years] & $63.0(21)$ & 34.0 & 78.0 & $61.0(19)$ & 40.0 & 71.0 & 0.8492 \\
\hline Weight [kg] & $66.0(21)$ & 52.0 & 96.0 & $64(19)$ & 50.0 & 99.0 & 0.5329 \\
\hline BMI & $25.2(21)$ & 19.8 & 33.2 & $24.7(19)$ & 20.4 & 34.0 & 0.2177 \\
\hline $\mathrm{ESR}[\mathrm{mm} / \mathrm{h}]$ & $14.5(18)$ & 2.0 & 60.0 & $16.0(17)$ & 3.0 & 60.0 & 0.9211 \\
\hline Rheumatoid factor [IU/ml] & $10.0(6)$ & 10.0 & 21.7 & $10.0(9)$ & 10.0 & 352.0 & 0.6900 \\
\hline NT-proBNP [pg/ml] & $153.5(14)$ & 46.0 & 871.0 & $195.0(13)$ & 12.0 & 684.0 & 0.9613 \\
\hline WBC $[K / \mu l]$ & $5.6(21)$ & 2.3 & 9.1 & $4.8(19)$ & 3.4 & 7.9 & 0.1013 \\
\hline Total protein TP [g/dl] & $6.6(7)$ & 6.4 & 10.5 & $6.3(5)$ & 6.2 & 6.7 & 0.0509 \\
\hline Albumins [\% TP] & $52.3(7)$ & 33.6 & 55.6 & $58.5(4)$ & 53.8 & 64.9 & 0.0233 \\
\hline $\mathrm{CRP}[\mathrm{mg} / \mathrm{l}]$ & $1.8(21)$ & 0.5 & 25.9 & $1.8(19)$ & 0.5 & 25.9 & 0.6837 \\
\hline $\mathrm{C} 3[\mathrm{~g} / \mathrm{l}]$ & $1.0(15)$ & 0.59 & 1.4 & $1.1(12)$ & 0.2 & 1.4 & 0.3539 \\
\hline $\mathrm{C} 4[\mathrm{~g} / \mathrm{ll}$ & $0.2(15)$ & 0.1 & 0.3 & $0.2(12)$ & 0.1 & 0.3 & 0.8643 \\
\hline CK [IU/I] & $70.0(3)$ & 36.0 & 81.0 & $67.5(10)$ & 13.4 & 262.2 & 0.8656 \\
\hline Disease duration [years] & $8.0(21)$ & 1.0 & 30.0 & 12.00 (19) & 2.0 & 30.0 & 0.3282 \\
\hline
\end{tabular}

SSC - systemic sclerosis, SIBO - small intestinal bacterial overgrowth, BMI-body mass index, ESR - erythrocyte sedimentation rate, WBC - white blood cells, $T P$ - total protein, CRP-C-reactive protein, C3, C4 - complement component 3, 4, CK-creatine kinase.

In SIBO assessment we used LHBT, which was the main diagnostic tool in two recent studies [30, 31]. However, most of the research groups used GHBT to diagnose SIBO in SSc patients [26-29, 32]. LHBT and GHBT show a comparable sensitivity in SSc patients [28, 31]. GHBT is characterized by $20-93 \%$ sensitivity and $30-86 \%$ specificity, whereas LHBT has $17-68 \%$ sensitivity and $44-86 \%$ specificity. Despite moderately better diagnostic performance of GHBT, we chose LHBT to avoid the underdiagnosis and undertreatment of patients with SIBO [41]. Furthermore, LHBT was suggested as a first-line diagnostic option by the Gastrolyzer manufacturer.

In our study the average SSc duration was longer in patients with SIBO by 4 years. Recent data set this difference on average of 3.7 years. We found no connection between SIBO and SSc subset, thus confirming the recent study outcomes. No connection between SIBO and the type of autoantibodies was found. Whereas some literature findings suggest less frequent occurrence of Scl-70 antibodies in $\mathrm{SIBO}[30,32]$. Our research reported only one relevant laboratory finding in SIBO patients concerning a higher serum albumin level, which hitherto remained unclear [31, 33]. The SSc gastrointestinal symptoms reported by the SSc patients with and without SIBO diagnosis did not differ significantly. The same outcomes were reported by Parodi et al., who explained this observation by the suboptimal LHBT sensitivity in SIBO diagnosis in SSC and probable underestimation of SIBO prevalence in SSC [30]. The lack of severe GI symptoms did not exclude SIBO diagnosis. Therefore, diagnostic procedures towards SIBO in SSC patients should be considered regardless of the presence of intense gastrointestinal symptoms. In other studies the prevalence of the following symptoms such as abdominal pain/discomfort, bloating, diarrhoea, constipation and abdominal tenderness was higher in SSC patients with SIBO diagnosis $[28,30]$.

In our research the eradication of SIBO was achieved in $73.3 \%$ of the patients. Most recent literature data estimated the efficacy of octreotide or ciprofloxacin at $100 \%$, of rifaximin at $73.3 \%$ and of norfloxacin and metronidazole at $52.4 \%$. Whereas the intermittent, rotating therapy with amoxicillin, ciprofloxacin and metronidazole suggested by EULAR from 2016 was associated with the eradication rate at a level of scarcely $43 \%[15,18,28,30$, $32,42,43]$. For the subject of our research we chose the evaluation of rifaximin in SIBO eradication due to short time of the therapy and good treatment tolerance. Up till now, only 2 studies assessed rifaximin efficacy in SIBO eradication in SSc patients. Parodi et al. introduced the same treatment regimen as we used and observed the identical eradication rate of $73.3 \%$. Whereas, Gemignani et al. beside rifaximin administered to the patients partially hydrolyzed guar gum obtaining 100\% eradication rate. Though, it is noteworthy that this therapy was introduced in only 12 patients $[27,30]$.

We observed a decrease in the incidence of all gastrointestinal complaints in SSc patients after rifaximin treatment during the second LHBT measurement. Statistically significant differences between two measurements were observed in case of constipation (57.1\% vs. $87.5 \%, p=0.0412$ ). In patients who achieved eradication of SIBO, previous studies report significantly improved diarrhoea, abdominal pain, discomfort and tenderness, 
nausea, emesis, bloating all significantly decreased [15, $27,29,35]$.

It is essential to establish the gastrointestinal involvement in SSc patients. The clinical assessment of digestive symptoms enables early detection and treatment of potential gastrointestinal malfunctions. The use of specific questionnaires helps to objectively monitor the gastrointestinal complaints impairment. Significantly frequent presence of SIBO among SSc patients indicates the necessity of introducing diagnostic test, which is noninvasive, cheap and accessible for the patients. These criteria are fulfilled by LHBT. Frequently, the gastrointestinal complaints of SSc patients remain similar regardless of SIBO presence. In this certain group of patients, the diarrhoea and malabsorption caused by SIBO can significantly worsen already existing difficulties with oral food intake and lead to malnutrition exacerbating the course of the disease. Therefore, we should consider performing LHBT even in patients with no severe gastrointestinal symptoms in order to avoid the delay of the diagnosis and treatment. Rifaximin turned out to be an effective, well-tolerated and short therapeutic regimen widely accepted by the patients. The eradication of the disease results in the decrease of gastrointestinal complaints in SSc patients and may significantly improve patient's quality of life and may reduce the risk of potential complications of a long-term disease.

This study had certain limitations. The small sample size could create bias. Further larger prospective studies are needed to confirm our reports.

\section{Acknowledgments}

We would like to express our special appreciation and thanks to Professor Wojciech Polkowski, by whose courtesy we were able to perform lactulose hydrogen breath tests with the Gastrolyzer.

\section{Conflict of interest}

The authors declare no conflict of interest.

\section{References}

1. Denton CP, Khanna D. Systemic sclerosis. Lancet 2017; 390: 1685-99.

2. Elhai M, Avouac J, Kahan A, Allanore Y. Systemic sclerosis: recent insights. Joint Bone Spine 2015; 82: 148-53.

3. Elhai M, Meune C, Avouac J, et al. Trends in mortality in patients with systemic sclerosis over 40 years: a systematic review and meta-analysis of cohort studies. Rheumatology 2012; 51: 1017-26.

4. Tyndall AJ, Bannert B, Vonk M, et al. Causes and risk factors for death in systemic sclerosis: a study from the EULAR Scleroderma Trials and Research (EUSTAR) database. Ann Rheum Dis 2010; 69: 1809-15.

5. Santosa A, Tan CS, Teng GG, et al. Lung and gastrointestinal complications are leading causes of death in SCORE, a multi-ethnic Singapore systemic sclerosis cohort. Scand J Rheumatol 2016; 45: 499-506.

6. Bures J, Cyrany J, Kohoutova D, et al. Small intestinal bacterial overgrowth syndrome. World I Gastroenterol 2010; 16: 2978-90.

7. Kumar S, Singh J, Rattan S, et al. Review article: pathogenesis and clinical manifestations of gastrointestinal involvement in systemic sclerosis. Aliment Pharmacol Ther 2017; 45: 883-98.

8. Miller JB, Gandhi N, Clarke J, McMahan Z. Gastrointestinal involvement in systemic sclerosis: an update. J Clin Rheumatol 2018; 24: 328-37.

9. Sakkas LI, Simopoulou T, Daoussis D, et al. Intestinal involvement in systemic sclerosis: a clinical review. Dig Dis Sci 2018; 63: 834-44

10. Jaeger VK, Wirz EG, Allanore Y, et al. Incidences and risk factors of organ manifestations in the early course of systemic sclerosis: a longitudinal EUSTAR study. PLoS One 2016; 11: e0163894.

11. Meier FM, Frommer KW, Dinser R, et al. Update on the profile of the EUSTAR cohort: an analysis of the EULAR Scleroderma Trials and Research group database. Ann Rheum Dis 2012; 71: 1355-60.

12. Miazga A, Osinski M, Cichy W, Zaba R. Current views on the etiopathogenesis, clinical manifestation, diagnostics, treatment and correlation with other nosological entities of SIBO. Adv Med Sci 2015; 60: 118-24.

13. Quigley EMM. The spectrum of small intestinal bacterial overgrowth (SIBO). Curr Gastroenterol Rep 2019; 21: 3.

14. Quigley EM. Small intestinal bacterial overgrowth: what it is and what it is not. Curr Opin Gastroenterol 2014; 30: 141-6.

15. Kaye SA, Lim SG, Taylor M, et al. Small bowel bacterial overgrowth in systemic sclerosis: detection using direct and indirect methods and treatment outcome. Br J Rheumatol 1995; 34: 265-9.

16. Erdogan A, Rao SS, Gulley D, et al. Small intestinal bacterial overgrowth: duodenal aspiration vs glucose breath test. Neurogastroenterol Motil 2015; 27: 481-9.

17. Marie I, Leroi AM, Gourcerol G, et al. Lactose malabsorption in systemic sclerosis. Aliment Pharmacol Ther 2016; 44: 1123-33.

18. Pittman N, Rawn SM, Wang M, et al. Treatment of small intestinal bacterial overgrowth in systemic sclerosis: a systematic review. Rheumatology 2018; 57: 1802-11.

19. van den Hoogen F, Khanna D, Fransen J, et al. 2013 classification criteria for systemic sclerosis: an American College ofRheumatology/European League against Rheumatism collaborative initiative. Arthritis Rheum 2013; 65: 2737-47.

20. LeRoy EC, Medsger TA Jr. Criteria for the classification of early systemic sclerosis. J Rheumatol 2001; 28: 1573-6.

21. Khanna D, Nagaraja V, Gladue H, et al. Measuring response in the gastrointestinal tract in systemic sclerosis. Curr Opin Rheumatol 2013; 25: 700-6.

22. McMahan ZH, Hummers LK. Gastrointestinal involvement in systemic sclerosis: diagnosis and management. Curr Opin Rheumatol 2018; 30: 533-40.

23. Gregersen H, Liao D, Pedersen J, Drewes AM. A new method for evaluation of intestinal muscle contraction properties: studies in normal subjects andin patients with systemic sclerosis. Neurogastroenterol Motil 2007; 19: 11-9.

24. Grace E, Shaw C, Whelan K, Andreyev HJ. Review article: small intestinal bacterial overgrowth: prevalence, clinical features, current and developing diagnostic tests, and treatment. Aliment Pharmacol Ther 2013; 38: 674-88. 
25. Frech TM, Mar D. Gastrointestinal and hepatic disease in systemic sclerosis. Rheum Dis Clin North Am 2018; 44: 15-28.

26. Fynne L, Worsoe J, Gregersen T, et al. Gastrointestinal transit in patients with systemic sclerosis. Scand I Gastroenterol 2011; 46: 1187-93.

27. Gemignani L, Savarino V, Ghio M, et al. Lactulose breath test to assess oro-cecal transit delay and estimate esophageal dysmotility in scleroderma patients. Semin Arthritis Rheum 2013; 42: 522-9.

28. Marie I, Ducrotte P, Denis P, et al. Small intestinal bacterial overgrowth in systemic sclerosis. Rheumatology 2009; 48: 1314-9.

29. Marie I, Leroi AM, Menard JF, et al. Fecal calprotectin in systemic sclerosis and review of the literature. Autoimmun Rev 2015; 14: 547-54.

30. Parodi A, Sessarego M, Greco A, et al. Small intestinal bacterial overgrowth in patients suffering from scleroderma: clinical effectiveness of its eradication. Am J Gastroenterol 2008; 103: 1257-62.

31. Savarino E, Mei F, Parodi A, et al. Gastrointestinal motility disorder assessment in systemic sclerosis. Rheumatology 2013; 52: 1095-100.

32. Tauber M, Avouac J, Benahmed A, et al. Prevalence and predictors of small intestinal bacterial overgrowth in systemic sclerosis patients with gastrointestinal symptoms. Clin Exp Rheumatol 2014; 32 (Suppl. 8): S82-7.

33. Rezaie A, Pimentel M, Rao SS. How to test and treat small intestinal bacterial overgrowth: an evidence-based approach. Curr Gastroenterol Rep 2016; 18: 8.

34. Scarpellini E, Giorgio V, Gabrielli M, et al. Prevalence of small intestinal bacterial overgrowth in children with irritable bowel syndrome: a case-control study. J Pediatr 2009; 155: 416-20.

35. Rana SV, Sinha SK, Lal S, et al. Small intestinal bacterial overgrowth in North Indian patients with celiac disease. Trop Gastroenterol 2007; 28: 159-61.

36. Rubio-Tapia A, Barton SH, Rosenblatt JE, Murray JA. Prevalence of small intestine bacterial overgrowth diagnosed by quantitative culture of intestinal aspirate in celiac disease. J Clin Gastroenterol 2009; 43: 157-61.

37. Tursi A, Brandimarte G, Giorgetti G. High prevalence of small intestinal bacterial overgrowth in celiac patients with persistence of gastrointestinal symptoms after gluten withdrawal. Am J Gastroenterol 2003; 98: 839-43.

38. Funayama Y, Sasaki I, Naito H, et al. Monitoring and antibacterial treatment for postoperative bacterial overgrowth in Crohn's disease. Dis Colon Rectum 1999; 42: 1072-7.

39. Klaus J, Spaniol U, Adler G, et al. Small intestinal bacterial overgrowth mimicking acute flare as a pitfall in patients with Crohn's disease. BMC Gastroenterol 2009; 9: 61.

40. Lin HC. Small intestinal bacterial overgrowth: a framework for understanding irritable bowel syndrome. JAMA 2004; 292: 852-8.

41. Saad RJ, Chey WD. Breath testing for small intestinal bacterial overgrowth: maximizing test accuracy. Clin Gastroenterol Hepatol 2014; 12: 1964-72.

42. Kowal-Bielecka O, Fransen J, Avouac J, et al. Update of EULAR recommendations for the treatment of systemic sclerosis. Ann Rheum Dis 2017; 76: 1327-39.

43. Haruma K, Wiste JA, Camilleri M. Effect of octreotide on gastrointestinal pressure profiles in health and in functional and organic gastrointestinal disorders. Gut 1994; 35: 1064-9. 